\title{
Lipid fluidity of triacylglycerol-rich lipoproteins isolated from copper-deficient rats*
}

\author{
BY CLAUDE MOTTA \\ Centre de Recherche en Nutrition Humaine de Clermont-Ferrand, Laboratoire de Biochimie, Hôtel \\ Dieu, 63000 Clermont-Ferrand, France
}

AND ELYETT GUEUX, ANDRZEJ MAZUR AND YVES RAYSSIGUIER $†$

Laboratoire des Maladies Métaboliques INRA, Theix 63122 St-Genès-Champanelle, France

(Received 19 April 1995 - Revised 6 July 1995 - Accepted 16 August 1995)

\begin{abstract}
Triacylglycerol-rich lipoproteins (TGRLP) were isolated from Cu-deficient and control rats. TGRLP from Cu-deficient rats appeared more fluid than those from controls as sensed by the fluorescence anisotropy of 1,6-diphenyl-1-3,5-hexatriene (DPH). This high fluidity was related to a low cholesterol: phospholipid ratio and high triacylglycerol content in these lipoproteins. TGRLP from $\mathrm{Cu}-$ deficient rats were more susceptible to in vitro peroxidation than lipoproteins from control rats as shown by the rate of diene conjugation. The damage induced by the peroxidation resulted in a more ordered state of the lipid fraction especially in lipoproteins from Cu-deficient rats. Thus, after in vitro peroxidation, TGRLP from Cu-deficient rats were more rigid than those from controls. These results suggest that $\mathrm{Cu}$ deficiency induces modifications in physicochemical properties of TGRLP which could affect their metabolism.
\end{abstract}

Copper: Lipoproteins: Peroxidation: Lipid fluidity

For many years, nutrition researchers have attempted to define the links between dietary trace elements and atherosclerotic cardiovascular disease. Severe $\mathrm{Cu}$ deficiency in rats results in a number of unfavourable changes that might contribute to cardiovascular risk and affect lipid metabolism (Lei, 1990). Hypercholesterolaemia is associated with an increase in apolipoprotein B plasma concentration (Nassir et al. 1993). A corresponding increase in plasma triacylglycerol-rich lipoproteins (TGRLP) containing apolipoprotein B $(\mathrm{VLDL}+\mathrm{LDL})$ occurs in $\mathrm{Cu}$-deficient animals and marked alterations in TGRLP composition have been described (Rayssiguier et al. 1993). Moreover, $\mathrm{Cu}$ deficiency increases the susceptibility of lipoproteins to in vitro peroxidation (Rayssiguier et al. 1993). Both chemical composition (Sola et al. 1990) and oxidative processes (Borchman et al. 1992) may affect the bulk fluidity of lipoproteins. This is of special importance because the metabolic fate of plasma lipoproteins is profoundly influenced by their physical properties (Deckelbaum et al. 1977). The present report describes the effects of $\mathrm{Cu}$ deficiency in rats on lipid fluidity of TGRLP and the influence of in vitro-induced oxidative modification of lipoproteins on their physical properties.

* A part of this study was presented at the 8th International Symposium on Trace Elements in Man and Animals (TEMA), Dresden, Germany, May 1993.

$\dagger$ For reprints. 


\section{MATERIALS AND METHODS}

\section{Animals and diets}

The Institute's guide for the care and use of laboratory animals was followed. Twenty-four weanling male Wistar rats (IFFA-CREDO, L'Arbresle, France) weighing 60 (SE 3) g were divided at random into $\mathrm{Cu}$-deficient and control groups (twelve rats per group). Rats were housed in wire-bottomed cages in a temperature-controlled room $\left(22^{\circ}\right)$ with a $12 \mathrm{~h}$ light-dark cycle and pair-fed on the appropriate diets for 6 weeks. The semi-purified diets contained $(\mathrm{g} / \mathrm{kg})$ : casein 200 , sucrose 650 , maize oil 50 , alphacel 50 , DL-methionine 3 , choline bitartrate 2, modified AIN-76 mineral mix 35, AIN-76A vitamin mix 10 (ICN Biomedicals, Orsay, France). Cupric carbonate was omitted from the AIN-76 mineral mix. The Cu concentrations of the diets were $0.6 \mathrm{mg} / \mathrm{kg}$ (deficient) diet and $7.5 \mathrm{mg} / \mathrm{kg}$ (control) diet. Fed animals were killed after being anaesthetized with sodium pentobarbital ( $40 \mathrm{mg} / \mathrm{kg}$ body weight given intraperitoneally). Blood was collected into heparinized tubes and plasma was obtained by low-speed centrifugation $(2000 \mathrm{~g})$. Packed cell volume was determined by centrifugation in a capillary tube system.

\section{Lipoprotein separation}

Equal volumes of plasma samples from two animals were pooled for lipoprotein separation. Ultracentrifugation was performed at $15^{\circ}$ in a Beckman L-5-50 ultracentrifuge (Beckman Instruments, Palo Alto, CA) with a Ti 50 rotor (Havel et al. 1955). EDTA (1 g/1) and butylated hydroxytoluene (BHT) $(4.4 \mathrm{mg} / \mathrm{l})$ were added immediately before lipoprotein separation. Samples were overlayered with $0.15 \mathrm{M}-\mathrm{NaCl}$ (density $1.006 \mathrm{~kg} / \mathrm{l}$ ) and chylomicrons were discarded following two centrifugations for $30 \mathrm{~min}$ at $12000 \mathrm{~g}$. To isolate the VLDL + LDL fraction, the plasma was adjusted to density $1.050 \mathrm{~kg} / 1$ with solid $\mathrm{KBr}$. Centrifugation was at $100000 \mathrm{~g}$ for $20 \mathrm{~h}$. The VLDL+LDL fraction was then washed by a further period of ultracentrifugation at the same density.

\section{Oxidation experiments}

Before oxidation experiments the purified lipoprotein fractions were dialysed against $0.01 \mathrm{M}$-phosphate buffer ( $\mathrm{pH} 7.4$ ) containing $0.15 \mathrm{M}-\mathrm{NaCl}$, which was made $\mathrm{O}_{2}$-free by vacuum-degassing followed by purging with $\mathrm{N}_{2}$. Suitable volumes from the dialysed solutions were diluted with phosphate-buffered saline (PBS) to obtain a final concentration of $0.5 \mathrm{~g} / \mathrm{l}$. Lipoprotein concentration was calculated from the mass of protein plus individual lipids. Oxidation experiments were performed as previously described (Esterbauer et al. 1989). Oxidation was initiated by addition of freshly prepared $\mathrm{CuSO}_{4}$ solution ( $5 \mu \mathrm{M}$ final concentration) at $37^{\circ}$. Oxidation of lipoproteins in vitro is accompanied by characteristic changes in chemical properties. Conjugated dienes develop in lipoproteins through the oxidation of polyunsaturated fatty acids (PUFA) with isolated double bonds to PUFA-hydroperoxides with conjugated double bonds (dienes) with a u.v. absorption maximum at $234 \mathrm{~nm}$. The kinetics of the oxidation of lipoproteins were monitored by the change in the $234 \mathrm{~nm}$ absorbance at $37^{\circ}$ (Esterbauer et al. 1989) on a spectrophotometer (Uvikon 820, Kontron, St-Quentin-en-Yvelines, France) and conjugated diene formation was expressed as the difference between the absorbance $5 \mathrm{~h}$ after and before in vitro oxidation.

\section{Polarization fluorescence studies}

Lipoprotein fluidity was assessed at $25^{\circ}$ by determining the anisotropy of fluorescence from the probe 1,6-diphenyl-1-3,5-hexatriene (DPH) (Ben Yashar \& Barenholz, 1991). Probe incorporation was accomplished by diluting $2 \mathrm{mM}-\mathrm{DPH}$ in tetrahydrofuran 1000 times into the aqueous lipoprotein solution before and $5 \mathrm{~h}$ after in vitro oxidation and incubating with 


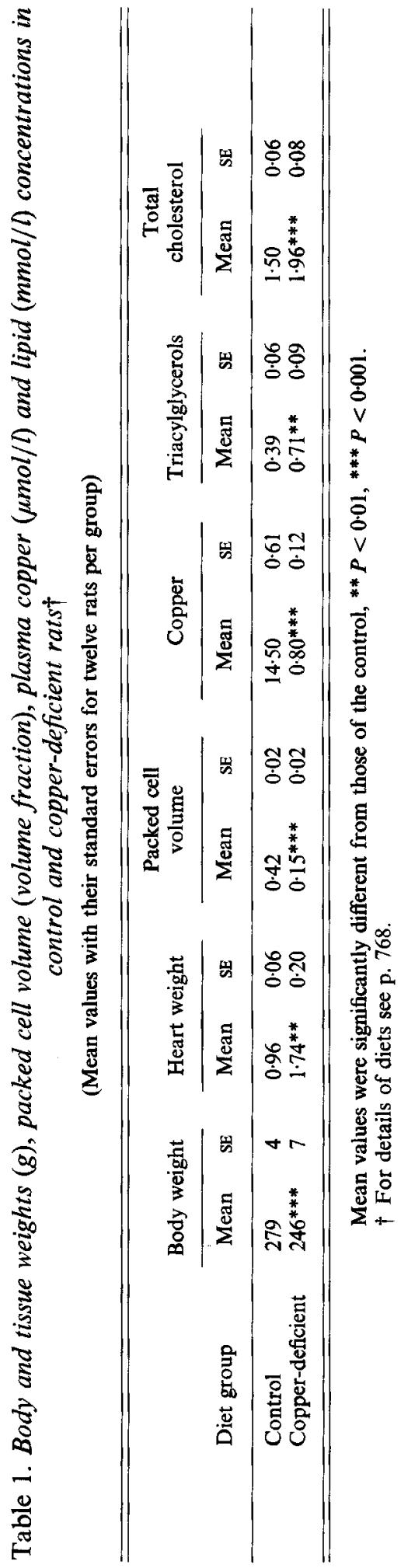


Table 2. Proportions of protein, triacylglycerols, cholesterol and phospholipids in $V L D L+L D L$ fractions $(\mathrm{mg} / \mathrm{g})$ in rats fed on a control or copper-deficient diet $\dagger$

(Mean values with their standard errors for six samples per group)

\begin{tabular}{|c|c|c|c|c|c|c|c|c|}
\hline \multirow[b]{2}{*}{ Diet group } & \multicolumn{2}{|c|}{ Protein } & \multicolumn{2}{|c|}{ Triacylglycerols } & \multicolumn{2}{|c|}{$\begin{array}{c}\text { Total } \\
\text { cholesterol }\end{array}$} & \multicolumn{2}{|c|}{ Phospholipids } \\
\hline & Mean & SE & Mean & $\mathbf{S E}$ & Mean & $\mathrm{SE}$ & Mean & SE \\
\hline Control & 114 & 6 & 518 & 17 & 186 & 9 & 181 & 7 \\
\hline Copper-deficient & 102 & 4 & $619 * * *$ & 12 & $104 * * *$ & 4 & 174 & 5 \\
\hline
\end{tabular}

Mean values were significantly different from those of the control, ${ }^{* * *} P<0.001$.

$\uparrow$ For details of diets see p. 768.

agitation at $25^{\circ}$ for $1 \mathrm{~h}$. Fluorescence polarization intensity was measured with a SEFAM spectrofluorometer (Vandoeuvre-lès-Nancy, France). In order to avoid any scattering problems, labelled and non-labelled samples were studied and the corrected anisotropy calculated (Gruneberger et al. 1982). With the amount of lipoproteins used, no correction was necessary. Moreover, the dilution method with the extrapolated final anisotropy led to the same conclusion (Lentz et al. 1979).

\section{Plasma and lipoprotein analysis}

Triacylglycerols (Biotrol, Paris, France), cholesterol and phospholipids (Biomérieux, Charbonnières-les-Bains, France) were determined in plasma and lipoprotein fractions by enzymic procedures. The protein concentration of the VLDL + LDL fraction was determined by a modified Lowry method using bovine serum albumin, fraction V (Sigma, L'Isle d'Abeau, France) as a standard (Markwell et al. 1978). $\mathrm{Cu}$ in plasma and diets was determined with a Perkin Elmer 560 atomic absorption spectrophotometer (Norwalk, CT, USA).

\section{Statistical methods}

Results are expressed as means with their standard errors. The statistical significance of differences between means was assessed by Student's $t$ test.

\section{RESULTS}

Rats fed on the $\mathrm{Cu}$-deficient diet had a lower body weight and lower plasma $\mathrm{Cu}$ concentration than those fed on the $\mathrm{Cu}$-adequate diet. In addition, $\mathrm{Cu}$-deficient rats were characterized by reduced packed cell volumes and enlarged hearts. Plasma cholesterol and triacylglycerol concentrations were significantly greater in $\mathrm{Cu}$-deficient rats than control animals (Table 1).

Marked alterations in the lipid composition of lipoproteins occurred in the $\mathrm{Cu}$-deficient rats. The proportion of triacylglycerols in the VLDL + LDL fraction was elevated. The proportion of cholesterol was markedly reduced whereas that of phospholipids was not significantly modified (Table 2). Consequently, $\mathrm{Cu}$-deficiency resulted in a decrease in the cholesterol: phospholipid ratio.

$\mathrm{Cu}$ deficiency substantially affected lipoprotein fluidity. VLDL + LDL anisotropy values were significantly lower in $\mathrm{Cu}$-deficient rats than in controls. The lipoproteins from the deficient rats were more susceptible to in vitro lipid peroxidation as shown by the rate of diene conjugation (absorption at $234 \mathrm{~nm}$ ). Lipid oxidation resulted in an increased 
Table 3. Effect of dietary copper on conjugated diene production ( $\Delta$ absorbance at $234 \mathrm{~nm})$ and anisotropy $(r)$ of $V L D L+L D L$ fractions $\dagger$

(Mean values with their standard errors for six samples per group)

\begin{tabular}{|c|c|c|c|c|c|c|}
\hline \multirow[b]{3}{*}{ Dietary group } & \multirow{2}{*}{\multicolumn{2}{|c|}{$\begin{array}{c}\text { Conjugated } \\
\text { dienes }\end{array}$}} & \multicolumn{4}{|c|}{ Anisotropy§ } \\
\hline & & & \multicolumn{2}{|c|}{ Unincubated } & \multicolumn{2}{|c|}{ Incubated } \\
\hline & Mean. & $\mathrm{SE}$ & Mean & $\mathrm{SE}$ & Mean & SE \\
\hline $\begin{array}{l}\text { Control } \\
\text { Copner-deficient }\end{array}$ & $\begin{array}{l}1.285 \\
1.851^{* *}\end{array}$ & $\begin{array}{l}0.048 \\
0.124\end{array}$ & $\begin{array}{l}0.125 \\
0.098^{* * *}\end{array}$ & $\begin{array}{l}0.003 \\
0.002\end{array}$ & $\begin{array}{l}0.143 \\
0.175^{* *}\end{array}$ & $\begin{array}{l}0.007 \\
0.004\end{array}$ \\
\hline
\end{tabular}

Mean values were significantly different from those of the control, ${ }^{* *} P<0.01, * * * P<0.001$.

$\dagger$ For details of diets and procedures, see pp. 768-770.

$\ddagger$ Conjugated diene formation was expressed as the difference between the absorbance at $234 \mathrm{~nm} 5 \mathrm{~h}$ after and before in vitro oxidation.

$\S$ Anisotropy values were determined before incubation and $5 \mathrm{~h}$ after in vitro oxidation.

anisotropy of lipoproteins $(P<0.05$ for lipoproteins from control rats and $P<0.001$ from $\mathrm{Cu}$-deficient rats). However, after in vitro peroxidation the lipoproteins from $\mathrm{Cu}$-deficient rats exhibited significantly higher anisotropy values compared with those obtained under the same conditions with lipoproteins from control animals (Table 3).

\section{DISCUSSION}

Several measurements, such as decreased body weight, increased heart weight and lower packed cell volume (Prohaska, 1990), are indicators of dietary $\mathrm{Cu}$ deficiency in addition to the reduced plasma $\mathrm{Cu}$ concentration. The mechanism by which $\mathrm{Cu}$ deficiency induces hyperlipaemia is not understood completely. Studies on hepatic apolipoprotein synthesis indicate that apolipoprotein B100 synthesis is increased in Cu-deficient rats (Nassir et al. 1993). Thus, the hyperlipaemia observed appears to be sustained by an increase in hepatic lipoprotein synthesis (Al-Othman et al. 1993). However, the possible contribution of defective uptake of plasma lipoproteins may also be involved in hyperlipaemia (Lei, 1990). Our results confirm previous observations that the lipid composition of VLDL + LDL from $\mathrm{Cu}$-deficient rats differs markedly from that of control rats (Rayssiguier $\mathrm{et} \mathrm{al}$. 1993). Results of the present experiment indicate that the overall fluidity of the lipoproteins as sensed by the fluorescence anisotropy of DPH is markedly enhanced in deficient animals. Wellestablished lipid modulators of the fluidity are known and especially the cholesterol:phospholipid ratio (C:PL) (Shinitzky, 1982). In Cu-deficient rats the C:PL ratio is drastically reduced and the triacylglycerol content increased, which strongly favours a high fluidity. In fact, cholesterol acts as an ordering molecule for the acyl chains of phospholipids and this results in restriction of movements of these chains; the low C:PL observed is consistent with the consequent effect on the bulk lipid fluidity (Shinitzky, 1982). Moreover, an increased content of triacylglycerols results in a more fluid and dynamic state of the core of lipoproteins (Berlin \& Young, 1983; Ben Yashar \& Barenholz, 1991). In the present experiment DPH was used to determine the core properties of lipoproteins. To determine if peroxidation affects the lipoprotein envelopes it would be useful to use trimethyl ammonium (TMA)-DPH (Ben Yashar \& Barenholz, 1991). Under normal conditions $\mathrm{Cu}$ does not occur in vivo in free form, being tightly bound to proteins (Parthasarathy \& Steinberg, 1992), whereas oxidation of lipoproteins in vitro proceeds at a high rate when the 
medium contains transition metal ions (Esterbauer et al. 1991). Our experimental model used the $\mathrm{Cu}$ oxidation process for lipoproteins. The conjugated diene values $5 \mathrm{~h}$ after in vitro oxidation confirmed that VLDL + LDL particles from deficient animals are more susceptible to oxidative damage than those of control rats. The aim of a previous study was to determine in more detail the time-course of lipoprotein oxidation (Rayssiguier et al. 1993). The lipoprotein fraction from Cu-deficient rats was readily oxidized as indicated by the short lag phase and the rapid increase in the rate of conjugated diene formation. In this previous experiment (Rayssiguier et al. 1993) lipid hydroperoxide and thiobarbituric-acidreactive substance measurements after the induction of peroxidation were in agreement with the greater formation of conjugated dienes. It is well known that lipid oxidation results in an apparently more ordered lipid organization (Richter, 1987). In fact, the oxidative process, throughout its development, reaches a final point leading to linkage between fatty acids, reducing the mobility of the chains (Richter, 1987). This reduction of acyl-chain movement affects the bulk fluidity of the particles. Our results from fluorescence polarization are fully in agreement with these kinds of oxidative damage. All the lipid organization results in an important rigidification of the lipid fraction as indicated by the DPH movements. However, the same oxidative process affects lipoproteins from control and deficient rats differently. The differences observed between anisotropy values for incubated and unincubated particles are markedly enhanced in $\mathrm{Cu}$-deficient rats compared with control ones. A more ordered state of lipoproteins from $\mathrm{Cu}$-deficient rats may depend on the more severe degree of oxidation of these lipoproteins (Richter, 1987). Major disturbances of antioxidant status occur in $\mathrm{Cu}$ deficiency and the role of several alterations in the composition of the TGRLP that may affect lipoprotein oxidation has been previously discussed (Rayssiguier et al. 1993). Moreover, changes in fatty acid metabolism have been described with the fatty acid unsaturation index usually being higher in $\mathrm{Cu}$-deficient rats (Cunnane, 1990). These changes would help to explain the increase in fluidity and peroxidation of TGRLP. The present study also suggests that the state of lipid order before oxidation may influence the susceptibility. A highly fluid lipid organization, as observed in the VLDL + LDL particles from Cu-deficient rats, could be more affected by an oxidative process and the lack of cholesterol content in TGRLP from Cu-deficient rats could favour more damaging oxidative processes. Increased levels of cholesterol in synthetic liposomes have a stabilizing effect which may in turn decrease the rate of peroxidation providing protection against lipid peroxidation (Smith, 1991; McLean \& Hagamen, 1992). Thus, the less ordered state of lipid particles, as shown in Cu-deficient rats, may allow better access of free radicals to peroxidation sites. However, results from the present experiments indicate that lipoproteins from $\mathrm{Cu}$-deficient rats are more fluid than those from controls following 6 weeks on experimental diets. So, it would be of interest to investigate if a longterm $\mathrm{Cu}$ deficiency results in decreased lipid fluidity related to enhanced lipid peroxidation in vivo. Modifications of lipoproteins in $\mathrm{Cu}$ deficiency as shown by increased susceptibility to peroxidation, and the resulting rigidification, may interact with the ability of receptors to recognize such modified lipoproteins (Parthasarathy \& Steinberg, 1992). The relationship between lipoprotein fluidity and atherogenicity remains to be elucidated. However, it has been suggested that the atherogenicity of lipoproteins increases as they become less fluid (Souter, 1978). The role of $\mathrm{Cu}$ as an antioxidant nutrient for cardiovascular health has recently been emphasized (Allen \& Klevay, 1994). Dietary $\mathrm{Cu}$ is low in the Western diet and low intake may affect several stages of atherosclerosis adversely (Allen \& Klevay, 1994). The present study suggests that the alterations of the physicochemical properties of lipoproteins in $\mathrm{Cu}$ deficiency may play an important role in their metabolic fate. 


\section{REFERENCES}

Allen, K. G. D. \& Klevay, L. N. (1994). Copper : an antioxidant nutrient for cardiovascular health. Current Opinion in Lipidology 5, 22-28.

Al-Othman, A. A., Rosenstein, F. \& Lei, K. Y. (1993). Copper deficiency increases in vivo hepatic synthesis of fatty acids, triacylglycerols and phospholipids in rats. Proceedings of the Society for Experimental Biology and Medicine 204, 97-103.

Ben Yashar, V. \& Barenholz, Y. (1991). Characterization of the core and surface of human plasma lipoproteins. A study based on the use of five fluorophores. Chemistry and Physics of Lipids 60, 1-14.

Berlin, E. \& Young, C. Jr (1983). Effects of fat level, feeding period and source of fat on lipid fluidity and physical state of rabbit plasma lipoproteins. Atherosclerosis 48, 15-27.

Borchman, D., Lamba, O. P., Salmassi, S., Lou, M. \& Yappert, C. (1992). The dual effect of oxidation on lipid bilayer structure. Lipids 27, 261-265.

Cunnane, S. C. (1990). Copper and long chain fatty acid metabolism. In Role of Copper in Lipid Metabolism, pp. 161-178 [K. Y. Lei, editor]. Boca Raton, FL: CRC Press Inc.

Deckelbaum, R. J., Shipley, G. G. \& Small, D. M. (1977). Structure and interactions of lipids in human plasma low density lipoproteins. Journal of Biological Chemistry 252, 744-754.

Esterbauer, H., Puhl, H., Dieber-Rotheneder, M., Waeg, G. \& Rabl, H. (1991). Effect of antioxidants on oxidative modification of LDL. Annals of Medicine 23, 573-581.

Esterbauer, H., Striegl, G., Puhl, H. \& Rotheneder, M. (1989). Continuous monitoring of in vitro oxidation of human low density lipoprotein. Free Radical Research Communications 6, 67-75.

Gruneberger, D., Haimovitz, R. \& Shinitzky, M. (1982). Resolution of plasma membrane lipid fluidity in intake cells labelled with diphenyl hexatriene. Biochimica et Biophysica Acta 688, 764-774.

Havel, R. J., Eder, A. H. \& Bragdon, J. M. (1955). The distribution and chemical composition of ultracentrifugally separated lipoproteins in human serum. Journal of Clinical Investigation 34, 1345-1363.

Lei, K. Y. (editor) (1990). Role of Copper in Lipid Metabolism. Boca Raton, FL: CRC Press Inc.

Lentz, B. R., Moore, B. M. \& Barrow, D. A. (1979). Light scattering effects in the measurement of membrane microviscosity with diphenyl hexatriene. Biophysical Journal 25, 489-494.

McLean, L. R. \& Hagamen, K. A. (1992). Effect of lipid physical state on the rate of peroxidation of liposomes. Free Radical Biology and Medicine 12, 113-119.

Markwell, M. A. K., Hass, S. M., Bieber, L. L. \& Tolbert, N. E. (1978). A modification of the Lowry procedure to simplify protein determination in membrane and lipoprotein samples. Analytical Biochemistry 87, $206-210$.

Nassir, F., Mazur, A., Sérougne, C., Gueux, E. \& Rayssiguier, Y. (1993). Hepatic apolipoprotein B synthesis in copper-deficient rats. FEBS Letters 322, 33-36.

Parthasarathy, S. \& Steinberg, D. (1992). Cell-induced oxidation of LDL. Current Opinion in Lipidology 3, 313-317.

Prohaska, J. R. (1990). Biochemical changes in copper deficiency. Journal of Nutritional Biochemistry 1, 453-461.

Rayssiguier, Y., Gueux, E., Bussière, L. \& Mazur, A. (1993). Copper deficiency increases the susceptibility of lipoproteins and tissues to peroxidation in rats. Journal of Nutrition 123, 1343-1348.

Richter, C. (1987). Biophysical consequences of lipid peroxidation in membranes. Chemistry and Physics of Lipids 44, $175-189$.

Shinitzky, M. (1982). Membrane fluidity and cellular functions. In Physiology of Membrane Fluidity, vol. 1, pp. 1-52 [M. Shinitzky, editor]. Boca Raton, FL: CRC Press.

Smith, L. L. (1991). Another cholesterol hypothesis: cholesterol as antioxidant. Free Radical Biology and Medicine $11,47-61$.

Sola, R., Baudet, M. F., Motta, C., Maillé, M., Boisnier, C. \& Jacotot, B. (1990). Effects of dietary fats on the fluidity of human high-density lipoprotein: influence of the overall composition and phospholipids fatty acids. Biochimica et Biophysica Acta 1043, 43-51.

Souter, A. (1978). Does dietary fat influence plasma lipoprotein structure? Nature 273, 11-12. 